\title{
LA GESTIÓN EDUCATIVA UNIVERSITARIA VENEZOLANA: UN PLANTEAMIENTO DESDE LA ACCIÓN TRANSCOMPLEJA
}

\author{
José Luis Yovera Yecerra * \\ https://orcid.org/0000-0002-5294-0008
}

RECIBIDO: Abril 2020 / ACEPTADO: Junio 2020 / PUBLICADO: Septiembre 2020

Como citar: Yovera Yecerra, José (2020). La gestión educativa universitaria venezolana: un planteamiento desde la acción transcompleja. Telos: revista de Estudios Interdisciplinarios en Ciencias Sociales, 22 (3), Venezuela. (Pp.541-550).

DOI: www.doi.org/10.36390/telos 223.05

\section{RESUMEN}

La gerencia educativa universitaria es trascendental para el desarrollo de políticas públicas que emergen desde la acción del ente encargado y desde la perspectiva de los actores involucrados, será prolifera de acciones que garanticen el logro académico, meta primordial de las instituciones universitarias que obedecen a políticas centradas en la formación del ciudadano capaz de afrontar tareas y trabajos específicos de acuerdo al perfil de egreso al cual se formó, sin embargo el trasfondo de dichos logros trasciende desde la interacción inter y transdisciplinar que el gerente debe propiciar para el logro satisfactorio de los objetivos prometidos, donde lo importante sea las contribuciones positivas al actor involucrado. El objetivo del articulo es de indicar aspectos resaltantes para la gestión educativa desde la acciones transcomplejas del transgerente, definido por Rodríguez (2017), se usó la Deconstrucción como transmétodo transcomplejo inédito de Rodríguez (2019). Es importante señalar que la interrelación que debe coexistir con las instituciones que son campo de aplicación de las carreras que se administran en las universidades, deben cooperar para la formación integral del estudiante y deben estar guiadas por el encargado de gerenciar los espacios académicos, permitiendo una interacción multidisciplinar que converja en la generación de acciones propicias para fomentar la acción pedagógica. Las características de una gestión transcompleja debe impulsar la formación desde los diferentes ámbitos de aplicación universitaria, a saber, la investigación y la formación permanente; la extensión universitaria y el compartir de saberes y la docencia con una visión transdisciplinaria.

Palabras Clave: Gestión Universitaria, Transdisciplinariedad, Acción Transcompleja, Transgerente.

\footnotetext{
* Postdoctorado en Políticas Publicas y Educación, Dr. En Educación, Esp. en Procesos Didácticos del nivel básico, Lcdo en Ciencias Matemáticas, Profesor de Matemáticas. Profesor de la Universidad Nacional Experimental del Yaracuy. Chivacoa- Venezuela. Correo electrónico: jyovera@uney.edu.ve
} 


\section{Venezuelan university educational management: an approach from transcomplex action.}

\section{ABSTRACT}

The university educational management is transcendental for the development of public policies that emerge from the action of the entity in charge and from the perspective of the actors involved, will be proliferated by actions that guarantee academic achievement, a primary goal of the university institutions that obey policies centered in the formation of the citizen capable of facing specific tasks and jobs according to the graduation profile to which it was formed, however the background of said achievements transcends from the inter and transdisciplinary interaction that the manager must promote for the satisfactory achievement of the promised objectives, where the important thing is the positive contributions to the actor involved. The objective of the article is to indicate outstanding aspects for educational management from the trans-complex actions of the trans-manager, defined by Rodriguez (2017), Deconstruction is used as an unpublished transcomplex method by Rodriguez (2019). It is important to point out that the interrelation that must coexist with the institutions that are field of application of the careers that are administered in the universities, must cooperate for the integral formation of the student and must be guided by the manager of the academic spaces, allowing a multidisciplinary interaction that converges in the generation of propitious actions to promote pedagogical action. The characteristics of a transcomplex management must promote training from the different fields of university application, namely, research and lifelong learning; The University Extension and the Sharing of knowledge and Teaching with a transdisciplinary vision.

Keywords: University Management, Transdisciplinarity, Transcomplex Action, Transcomplex manager.

\section{Introducción}

El crecimiento vertiginoso de la modernización desde el punto de vista tecnológico, ha influido en el desarrollo de mejoras en la educación de los diferentes niveles, esto permite generar reflexiones inmediatas sobre la forma de adaptación a esta sociedad del conocimiento, y de cómo generar innovaciones desde las universidades para convivir y estar al día con lo actual; una acción multiparadigmática, debe surgir de los actores involucrados y más aún de aquellos encargados de maniobrar los objetivos propios de la academia que van dirigidos a la promoción del conocimiento desde la diversidad de métodos que propicien el aprendizaje requerido en las diferentes aplicabilidades existentes en las carreras universitarias ofertadas, con miras a establecer los vínculos que la sociedad de la información requiere y exige para la interacción universidad-sociedad.

El objetivo del presente artículo va dirigido a indicar aspectos resaltantes para la gestión educativa desde la acciones transcomplejas del transgerente, es decir, proporcionar ideas constructivas de la gerencia educativa universitaria venezolana desde las tres actividades de trabajo asociados a las universidades como lo son la docencia, investigación y extensión, desde el accionar transcomplejo que permitan describir el contexto epocal y fenoménico en que 
desarrolla el devenir social-educativo que circunscriben al crecimiento intelectual, tecnológico, investigativo, productivo de las universidades del Sur con particularidad en Venezuela, para tal fin, se aplicó La Deconstrucción de Rodríguez (2019), como transmétodo transcomplejo.

La visión de las universidades en Venezuela debe estar dirigida en la promoción de actividades propias de la docencia que colinden en la generación de actividades creativas, innovadoras, intelectuales que evidencien la calidad de educación suministrada, debe ir de la mano con la convergencia de las unidades curriculares propias de cada carrera para trascender en la interactividad transdisciplinar, donde diversos puntos de vistas direccionen su aplicabilidad particular a una acción general, dando aportes importantes en la concreción de un fin común tal como lo sugiere Toffler (1996, p.12), cuando "reafirmaba la necesaria adaptabilidad de las organizaciones educativas a los diferentes cambios, como condición para la supervivencia" con la visión interactiva del planeta y sus diferentes contextos; este planteamiento debe ir de la mano con la investigación, donde la intervención de espacios de reflexión, discusión, planteamientos, formulación, interpretación, promoción y divulgación sean reiterativas y afianzadas en el espacio-tiempo dando paso al reconocimiento de líneas de investigación ante la sociedad del conocimiento y ser referentes inéditos e irrefutables.

La extensión universitaria como garante de la interacción universidad-comunidad, debe abrir los canales de discusión y concreción de avenencias que incidan en el complemento del aprendizaje que requiere el estudiante universitario, impulsando una visión crítica de su campo de aplicación y conviviendo en su futuro campo laboral, estableciendo parámetro de discusión en los diferentes ambientes de aprendizajes, propiciando la generación de conocimiento contextualizado tomados de la realidad existente trayendo esto una actualización al programa que muchas veces está diseñado con un fin perpetuo y no busca incorporar nuevas estrategias ni contenidos acorde avanza la educación que en lo actual y global tiene comportamientos exponenciales.

Estas acciones deben ser guiadas con firmeza y cordura, tratando de superar aspectos burocráticos complejos basados en redes intersubjetivas, que retrasan la concreción de los objetivos planteados en el nivel universitario; es preponderante tener claro las vías que propicien resultados satisfactorios y la intervención de la diversidad como se propone en la transcomplejidad, permite que los espacios y momentos proyectados como logros no se interrumpan y descuelle la universalización, producción y transmisión del conocimiento que influyan en la concepción de la institución como espacio de fomento de sujetos pensantes con visión crítica ante la comunidad del saber.

Bajo esta perspectiva el gerente emergente debe tener sosegado cada aspecto antes mencionado y debe iniciar una búsqueda amplia y desinteresada de acciones que confluyan en sintetizar las dificultades, Rodríguez (2017, p.119), plantea una forma de gerencia que denomina transgestión o gerencia transcompleja, concibiéndola vinculada con la ética. Sobre esta temática, la autora puntualiza:

"Se construye desde una práctica gerencial y docente, desde la realidad vital, desde la vida cotidiana, de la necesidad de saber, cómo actuar en cada situación concreta... Esto, irrefutablemente implica reflexión, sensibilidad, argumentación, desarrollo de hábitos. Requiere además de una profunda renovación de los paradigmas anclados a la gerencia en torno a cómo, cuándo y para qué gerenciar". 
Desde esta perspectiva, la ética se constituye en el principal valor, que requiere además del uso de las capacidades personales y fundamentos morales para el ejercicio de una gerencia consciente y reflexiva, aunado a esto la acción gerencial debe ir de la mano con la influencia ecosófica, conocida como el arte de convivir en el planeta (Rodríguez 2019, p. 6), interpretando su acción y su influencia en preservar el planeta, promocionar actividades que incidan en el quehacer cotidiano de los actores involucrados, incorporando esta idea en la transmodernidad como lo plantea Rodríguez (2019, p. 7), "la transmodernidad en plena consideración respalda el carácter ecosófico de las investigaciones transcomplejas".

La Transcomplejidad como lo plantea Rodríguez (2017, p. 120), como "forma de pensamiento presente en la reconstrucción y deconstrucción de las realidades complejas, interconectadas" hace a un lado el mito de que los actores involucrados en las universidades no se conviertan en sujetos colectivos de pensamiento con capacidad de generar innovaciones acordes a la emergente universalidad, debido a la existencia de visiones individualistas que conllevan a la adaptación de un solo pensamiento, que va orientado a una sola dirección.

Es importante señalar que, estar inmerso en la transcomplejidad es buscar aquello que está entre las disciplinas, a través de las disciplinas, más aún, más allá de las disciplinas permitiendo una interacción mucho más compleja lo que nos hace ver a la educación como una manera diferente de cohabitar en la cotidianidad actual. Es por ello que la gerencia universitaria desde una acción transcompleja en Venezuela, nos hace repensar en la forma de coexistencia de las diferentes disciplinas con la docencia, la investigación y la extensión universitaria con una perspectiva transmoderna.

En este sentido González (2013, p. 73), considera que la vinculación complejidad y transdisciplinariedad "responde a procesos de transformación que rompe los esquemas estables de cultura, sociedad, ciencia y todo lo que el ser humano cree controlar en su visión lineal, la respuesta es compleja y transdisciplinar" considerando además la incorporación del mundo fenoménico cambiante a cada instante, produciendo espacios de incertidumbre y azar propio de la complejidad esto se argumenta mediante lo dicho por Morín (2005, p. 3),"somos complejos, "híper-complejos" y más aún metacomplejos, donde aparece la incertidumbre, la angustia y el desorden como parte de su ser y existencia".

Pero que sucede cuando el sujeto toma las dos formas de ver el mundo; por un lado aquella situación compleja, y además toma conciencia de lo que él piensa, aprende o conoce, este proceso como tal favorece que el sujeto se apropie de la naturaleza en una cosmovisión metacompleja, es decir un pensamiento metacomplejo que caracterizamos según González (2009, p. 5), "como aquella forma de ver el mundo donde el observador toma conciencia de la realidad compleja", de esta manera el gerente debe activar las acciones que trasciendan en el logro de los propósitos de la educación universitaria con una visión planetaria amplia "sin perder los contextos socioculturales" (Rodríguez, 2019, p. 7), rompiendo esquemas coloniales anclados en tradicionalismo gerencial donde las decisiones van dirigidas unidireccionalmente.

En torno a ello en el presente artículo se considera presentar desde una perspectiva diferente la Gestión Educativa Universitaria Venezolana mediante acciones transcomplejas acordes a un proceso educativo actual, lleno de nuevas tendencias tecnológicas, amplitud global, incorporación e inclusión de las universidades venezolanas en una nueva visión social, se presentan elementos transformadores desde cada espacio estructural de la universidades Docencia, Investigación y Extensión Universitaria, donde se hacen deconstrucciones importantes en referencia a los elementos mencionados, en primer lugar se describe el 
transmétodo transcomplejo aplicado en la investigación, el segundo apartado se describe el rol de la docencia universitaria venezolana con perspectiva transdisciplinaria, en la tercera parte se muestra una deconstrucción para la investigación universitaria en Venezuela, como una oportunidad de formación permanente, se hace un análisis a la interactividad universidadsociedad, y por último se presentan las conclusiones que surgen de la deconstrucción que a su vez se convierten en puntos de partida para proseguir en la investigación.

\section{La Deconstrucción de Rodríguez (2019) como Transmetodología Transcompleja.}

Para indagar acerca de la Gestión Educativa Universitaria Venezolana y realizar un planteamiento desde la acción transcompleja, deconstruyendo el proceder gerencial anclado a la intervención tradicional burocrática, para ello se utilizó la Deconstrucción como Transmetodología Transcompleja inédita de Rodríguez (2019), en lo que sigue se indicará los aspectos meta-teóricos que regirá el estudio, iniciando por el transparadigma, el cual está inmerso en la Transcomplejidad, pues se trata de ir más allá -significado de trans- en la teoría de la investigación. En el transmétodo la Deconstrucción Rodríguez (2019, p.43) establece que "como transmétodo de investigación es libre al máximo, anti-dogmática, no tiene ninguna transmetodología fija, su objetivo es debilitar el pensamiento filosófico occidental, destruir las concepciones colonizantes en todas sus formas y significados".

Esta perspectiva conduce a repensar la gerencia educativa universitaria la cual debe erigir un nuevo planteamiento transmoderno, que sea abierto, pleno, complejo, cambiante a nuevas orientaciones, rompiendo esquemas burocratizados, alentando el bienestar de los actores involucrados en la universidad y que converjan en promover las diferentes acciones de la institución desde la docencia, investigación y extensión universitaria, unidas al pensamiento complejo como lo establecen Andrade, Leguizamo y Vergara (2018, p. 497), "el pensamiento complejo es una propuesta-desafío-invitación de reforma al pensamiento reduccionista que limita los saberes a circularidades"

Es importante acuñar esta investigación al proyecto de la Transmodernidad, ya que se debe revisar la Gerencia Universitaria Venezolana con una amplitud visionaria que permita generar un pensamiento abierto, profundo, crítico, que propicie aperturas que no cierran si no que vuelven con una mayor carga de conocimiento por indagar, que genere espacios de discusión incorporando intervenciones decoloniales, al respecto Rodríguez (2018, p. 434), que desde "su papel de formación de ciudadanos críticos, responsables, tolerantes a las diferencias culturales; pero amantes de nuestra inmensa diversidad de patrimonios naturales y culturales, muchas veces desconocidos". Indica en romper acciones ajustadas a viejos criterios gerencistas, donde el pensamiento que prevalece es el de quien está en la cúspide de la pirámide y aguas abajo se hace cumplir su voluntad.

Es importante reseñar el hecho de que incursionar los trabajos en la Transmodernidad que según Rodríguez (2020, p.61), "van dejando de lado el debate cualitativo-cuantitativosociocrítico y sin excluirlos va como un proceso complejo y transdisciplinario de construcción y reconstrucción del conocimiento", por su parte el transmétodo utilizado es una irreverencia al estudio sistemático amparado en patrones guías que propician un inicio, desarrollo y cierre sin detenerse a observar que cambios se generan en el desarrollo que deben mejorarse al inicio, y que un cierre nunca queda culminado más aún abre nuevas inquietudes, al respecto Rodríguez 
(2020), manifiesta "con la deconstrucción el autor estará en un "ir y venir que va a la criticidad; pero también a la reconstrucción".

Esta afirmación encaja en la idea transcompleja de de-construir y re-significar la gerencia educativa universitaria con giros decoloniales guiados por las ideas de Rodríguez (2016, p. 134), quien se refiere a que "el giro decolonial como una propuesta problemática y divergente con las diversas formas de comprender la modernidad".

\section{La Docencia Universitaria: una visión transdisciplinaria.}

La educación se ha convertido en un excelente objeto de estudio e investigación, las diferentes maneras de pensar y de repensar la realidad existente en los espacios educativos que buscan cambiar su acción para mejorar los resultados y promover la calidad educativa. Dewey $(1998$, p. 53) indicó que "el proceso educativo es un proceso de reorganización, reconstrucción y transformación continua", más aún debe incluir al individuo en las actividades complejas que acarrea el día a día, las cuales están impregnadas de problemas cotidianos de toma de decisiones de múltiples opciones donde la solución viable no es única ni lineal, al contrario surge de las condiciones que la persona ha establecido como conocimiento previo y de alguna manera generó un resultado satisfactorio, sin embargo no es garantía que al aplicarlo nuevamente sea satisfactorio, pues la existencia de situaciones multivariables acarrea diversas formas de mirar las situaciones y generar la solución más viable.

Lo transdisciplinar proporciona diferentes puntos de vista a una misma situación donde la incorporación de diferentes características que aporten a generar mejoras al aprendizaje, mediante planteamientos diferentes desde la aplicabilidad de una realidad que genere un conocimiento. Este planteamiento incorporado a la docencia universitaria permite la concurrencia de diferentes y diversos elementos que permiten la incorporación de múltiples aplicaciones y que el pensamiento del estudiante sea profundo, divergente, hologramático y permita incorporar en su intelecto valores educativos, de conciencia cognitiva de los diferentes procesos a los cuales está siendo sumergido.

La vertiginosa crecida de la globalidad en lo actual, hace repensar el proceder educativo donde convergen multitud de elementos, múltiples y variadas interacciones en procesos en los que el dinamismo es constante, esta globalidad sostiene que lo social ha generado una estructura modélica que hace establecer cambios paradigmáticos entorno a situaciones que ameriten solución a priori, es decir se establece una hiperinteracción sobre eventos sociales que inciden directamente en la educación. González (2013, p. 81), considera que "la injusticia social y la insostenibilidad ecológica presentes en la actualidad reclaman la construcción colectiva de nuevas formas de sentir, valorar, pensar y actuar en los individuos y en las colectividades", esto repercute en la idea inicial de un proceder distinto de la educación universitaria, que no aísla la influencia, reflexión y valoración de dimensiones ecosóficas desde lo social, político, económico, cultural y moral de la sociedad.

La complejidad como lo establece Morín, Ciurana y Motta (2003, p.14), indica que esta es "como un tejido de constituyentes heterogéneos inseparablemente asociados. Es la dialógica orden/organización. Supone temporalidad, no lineada, aleatoriedad, auto - organización, probabilidad para extraer nueva información", esto nos indica que la visión gerencial desde esta perspectiva debe ir contra dificultades, estableciendo parámetros que permitan el fomento de la educación desde sus diferentes vertientes, incidiendo de manera positiva. Desde este punto de vista la complejidad es una concepción distinta del hombre como sujeto que estudia la realidad 
desde una perspectiva dialógica. Una frase importante que indica Morín (2005, p. 32) es que la complejidad "es una ciencia del hombre y para el hombre. Es una aventura de acción cotidiana con vocación transdisciplinar, donde los diferentes aspectos del saber están en constante interacción y complementariedad".

La educación universitaria debe planificarse para asumir diferentes aspectos que involucre la investigación y la interacción de la universidad con la sociedad, teniendo presente que la producción de conocimiento está sustentada en lo social, cultural, político, académico, institucional y es allí donde el gerente educativo debe direccionar sus esfuerzos para lograr incorporar al estudiantado en la realidad emergente actual que impone mecanismos de interactividad social, cibernética, técnico y tecnológico, desde visiones complejas para establecer hechos de impacto social. En lo que una docencia universitaria de calidad debe incidir en la integralidad de las disciplinas que coadyuven en la adquisición de un conocimiento digerible desde distintas posiciones eliminando la unidireccionalidad que en muchos casos las unidades curriculares direccionan, siguiendo un plan eliminando la interacción docente-estudiante.

\section{La Investigación Universitaria, una oportunidad de formación permanente.}

La investigación universitaria es la cara frontal de toda institución educativa y da conocer los procesos que surgen en los espacios académicos, a diario se produce una investigación que nace desde una dificultad presente, o simplemente por una visión distinta a la que comúnmente se viene presentando la cotidianidad, es importante establecer parámetros de gestión investigativa, que produzca alimento intelectual para la sociedad del conocimiento. Las políticas gerenciales deben establecer vías, medios y metodologías para fomentar la investigación en las instituciones educativas universitarias y no limitarse a la producción que realizan los estudiantes para la obtención de un grado, si no que emerjan investigaciones producto de esa interacción cotidiana impregnada de complejidad.

Es un hecho que existe cierta renuencia respecto a realizar investigación mientras se está dictando una cátedra, pero el solo hecho de preparación de cada unidad de clase, la planificación de las actividades evaluativas, se convierte en un proceso investigativo, el detalle es la direccionalidad de la misma, Rojas y Ruiz (2001, p. 17), manifestaron lo siguiente respecto a la dualidad docencia-investigación "Resulta impactante que la enseñanza y la investigación estén tan separadas. Normalmente, los investigadores enseñan a tiempo parcial, pero los docentes no hacen investigación, además las estructuras de investigación y las de enseñanza casi siempre están separadas." Esto es un hecho que ha calado y mantenido en diferentes estructuras educativas que se limitan a realizar investigación en ocasiones apremiantes (trabajos de grado, ascensos o estudios de postgrado) y en reiteradas ocasiones van dirigidas a actividades fuera de las líneas de la carrera a la cual está suscrito dentro de la universidad.

La acción del gerente transcomplejo radica en la manera de incitar al proceso investigativo, estableciendo e impulsando espacios de investigación, divulgación y reproducción de hechos problémicos que requieran solución, punta de lanza de toda investigación, todo esto debe inducir a que los productos encontrados estrechen la relación entre los desarrollos disciplinarios y la transferencia en el recinto educativo de los adelantes transcendentales sin dejar a un lado la experiencia pedagógica. Su acción debe ser de gran impacto para que el renombre de las instituciones educativas prevalezca por encima de ínfulas individuales que acrediten a una sola persona; promoviendo el interés investigativo, no solo para ir escalando en la estructura 
universitaria sino para tener reconocimiento en la estructura del saber, proyectando tanto su nombre como el de la institución a la cual se suscribe.

Es importante mencionar que la investigación siempre ha permitido el crecimiento académico que surge de diversos factores adversos al proceder académico cotidiano y que produjo una incertidumbre ante un hecho circunstancial y que produce un lecho investigativo, esto permite al docente involucrase en actividades que le permita convivir entre la docencia y la investigación sin descuidar ninguno de los dos actos, el gerente debe promover la incorporación y la distribución de los espacios para la buena coexistencia, esto en afinidad con lo que establece Reyes (2017, p. 12), "Dentro de la gestión educativa, lo más importante es generar y apoyar procesos de cambio, donde los actores de estos procesos sean los que tomen en sus manos la decisión de transformar, desde los mismos actos de gestión". En este orden de ideas Rojas (2011, p. 3), considera que "las universidades tienen como parte de su misión el describir y producir explicaciones sobres las realidades, y más importante, contribuir a las transformaciones sociales que conduzcan a eliminar formas de dominación y despojo".

La gerencia transcompleja y la investigación deben complementarse para lograr objetivos de creación intelectual que confluyan con el realce de las instituciones universitarias bajo la perspectiva compleja y transdisciplinaria, con perspectiva multiparadigmática, en este sentido Balza (2006, p. 21), complementa la idea al explicar que la transdisciplinariedad "constituye una epistemología emergente, que mediante sus métodos particulares como modos de conocer, trasciende los límites disciplinarios del conocimiento, para abrirse paso sobre lo transcultural en forma transversal y compleja a través de la imaginación creadora del ser humano" Es concluyente el hecho que el planteamiento idóneo de la investigación de la mano de la gerencia debe inducir la construcción deconstrucción y reconstrucción dialéctica y dialógica del conocimiento en un ciclo continuo imparable.

\section{La Extensión Universitaria, un espacio idóneo para cohabitar universidad-sociedad.}

Dentro de la interactividad de las universidades, es relevante mencionar la relación existente con la realidad aplicable a cada una de las carreras vistas en las diferentes instituciones, esa relación es de vitalidad para el desarrollo de las diferentes actividades académicas, en este sentido, se establece que la Extensión Universitaria es una situación académica cuya visión es de interacción indisoluble con la sociedad y en particular con las diferentes comunidades tanto sociales como científicas e industriales para el fortalecimiento de la gnosis crítica con intención de mejorar la conciencia y el nivel social desde diferentes puntos vistas, bien sea en lo económico y cultural de las mismas.

Bajo esta perspectiva, la gerencia debe promocionar las actividades que se producen en cada instituciones mediante la divulgación continua, reuniones vinculantes directamente con las comunidades de aplicación respecto a cada carrera que se administre en la universidad, aunque en las políticas públicas venezolanas existe la estructura del Servicio comunitario, este se ha dedicado a realizar una actividad que muchas veces carece de profundidad académica y los logros son superficiales, ya que el interés es cumplir un número de horas para el grado más que aprovechar el espacio para la reflexión y discusión de aspectos vistos en los ambientes de aprendizaje.

Es por ello que el gerente educativo debe establecer acciones que garanticen el desenvolvimiento de los actores involucrados con la comunidad al respecto Briceño $(2009$, p. 5) reseño que "grandes transformaciones científico-tecnológicas y socio-culturales, se hace 
indispensable el rol mediador que debe jugar la extensión universitaria como factor de vinculación entre la universidad, los cambios del entorno y viceversa". Esta reflexión hace pensar la interactividad efectiva desde situaciones de incertidumbre que catalizan espacios de discusión social y compromete el real desenvolvimiento de los actores involucrados en la toma de decisiones idóneas.

La extensión universitaria debe presentar un espacio apto para coexistir la universidad y la comunidad, promoviendo acciones que le permitan a los estudiantes y a los docentes establecer verdaderos resultados que surgen de un problema, que es tan común y que desde los espacios de la academia son vistos con menos impacto e importancia, dando un valor agregado cuando la aplicabilidad permite resolver una situación compleja que involucra a muchos y su solución permite varias cosas, la primera bienestar a la comunidad estableciendo los verdaderos objetivos de la relación universidad-comunidad, segundo la adquisición del conocimiento a través de la experiencia directa, tercero la solución de realidades complejas que generalmente permiten la generaciones de subsituaciones que surgen del problema general y a su vez genera un problema.

\section{A manera de Conclusión}

En esta investigación transmétodica con la deconstrucción con cabida transcompleja se ha analizado la Gerencia Educativa Universitaria Venezolana, dando respuesta al objetivo de la investigación que subyace en indicar aspectos resaltantes para la gestión educativa desde las acciones transcomplejas del transgerente, se considera el cierre de la investigación que se convierte en una apertura, considerando lo siguiente:

Los fines de la gerencia educativa universitaria amparados en la acción transcompleja son de establecer los diversos espacios de reflexión, discusión y divulgación mediante los diferentes entes suscritos como campos de aplicación laboral, así como de comunidades que conviven en situaciones apremiantes que son espacios de querellas y su solución imbrican diferentes acciones que son disertadas en los ambientes de aprendizajes pero no siempre con perspectivas generalizadas.

La convergencia de los tres elementos que radican en el desenvolvimiento de todas las universidades, docencia, investigación y extensión es de vital importancia, ya que desde cada espacio la universidad producirá y el transgerente se encargará de establecer los vínculos que permitan mantener la triada en total producción, sin atropellarse unos con otros, permitiendo la inclusión de diversos puntos de vistas donde las diferentes disciplinas aporten significativamente, promoviendo el crecimiento personal y profesional de los involucrados, estrechando los lazos confraterno con las comunidades donde la misma sociedad genere situaciones donde la acción transcompleja permita el subterfugio a situaciones apremiantes, apoyados por la propia sociedad en general.

La Transgerencia debe motivar la promoción de actividades de divulgación científica desde los diferentes medios, tales como Congresos, Simposios, Jornadas, Reuniones, Presentaciones y propiciar espacios para la publicar los avances prácticos que surgen del proceder educativo como Revistas, inmersos en la realidad universitaria, pensar y repensar el proceder académico favoreciendo la academia donde los actores involucrados transmitan y trasciendan a favor de la Universidad. Particularmente la trangerencia transciende en la acción docente, encargado de propiciar aprendizajes, gerenciar en los espacios de formación, promover ambientes 
contextualizados, enaltecer sus ideas para que confluyan en el rescate de lo cultural, tradicional, enalteciendo el debate critico, decolonial, ecosófico.

La gerencia transcompleja y la investigación deben complementarse para lograr objetivos de creación intelectual que confluyan con el realce de las instituciones universitarias bajo la perspectiva compleja y transdisciplinaria, con perspectiva multiparadigmática, repensando la acción investigativa abriendo aristas que permitan generación de teorías y metateorías, proyectos factibles, mejoras tecnológicas y/o prototipos que den impulso a emprendimientos sólidos que emerjan del intelecto producido en los espacios de debate y discusión educativa.

\section{Referencias Bibliográficas}

Andrade, Leguizamo y Vergara (2018). Educación para la resistencia, una aproximación desde la complejidad, Revista Kavilando.Org. Volumen 10 №2, Colombia (Pp. 495-508)

Balza, Antonio. (2006). Pensar la investigación educativa y el aprendizaje desde la perspectiva de la transdisciplinariedad. 3er. Simposio de Postdoctorado en Ciencias de la Educación. San Joaquín de Turmero, Venezuela.

Briceño, Zaida. (2009). La extensión universitaria bajo la visión de la transcomplejidad, Revista Paradigma. Volumen 12 №2 Venezuela. (Pp.4-20)

Dewey, Jhon. (1998). Educación y Democracia. Editorial Gedisa. España.

González, Juan M. (2013). Aula mente social. Pensamiento transcomplejo. Tomo III. Editorial Mejoras, Colombia.

Morín, Edgar. (2005). Introducción al Pensamiento Complejo. UNESCO. Francia.

Morín, Edgar., Ciurana, Edgar. y Motta, Raúl. (2003). Educar en la era planetaria. Editorial Gedisa, España.

Reyes, Luis. (2017). Un modelo teórico de la acción tutorial en el postgrado. Tesis de grado. Universidad Rafael Belloso Chacín (URBE). Maracaibo, Venezuela.

Rodríguez, Abdiel (2016) El giro decolonial del siglo XXI. Revista Ensayos Pedagógicos Volumen XI Nº 2. Panamá. (Pp. 133-158)

Rodríguez, Milagros (2017) La Transgestión Educativa Transparadigmática en Venezuela.

Revista Praxis Investigativa Volumen 9 N$^{\circ} 17$ México. (Pp.118-129).

Rodríguez, Milagros. (2018). La Educación Patrimonial y la formación docente desde la transcomplejidad. TELOS. Revista de Estudios Interdisciplinarios en Ciencias Sociales, Volumen $20 \mathrm{~N}^{0} 3$, Venezuela. (Pp. 431-449).

Rodríguez, Milagros. (2019). Deconstrucción: un transmétodo rizomático transcomplejo en la transmodernidad. Sinergias educativas, Volumen. 4, № 2, Ecuador. (Pp. 1-13).

Rodríguez, Milagros. (2020).Miradas Transcomplejas de la Díada: Educación Matemática Crítica-Antropoética. Praxis Educativa ReDIE, Volumen 12, México (Pp. 58-76).

Rojas, Raúl (2011) Una mirada Transcompleja de la actualización e innovación de la Educación Universitaria apoyada en el uso de las tecnologías de información y comunicación. XII

Congreso Internacional de Teoría de la Educación. Barcelona, España.

Rojas, Raúl. y Ruiz, Antonio. (2001). Vínculo Docencia-Investigación para una formación integral. Plaza y Valdés Editores, México.

Toffler, Alvin. (1996). La tercera Ola. (Sexta edición). Editorial Paidós. EEUU. 